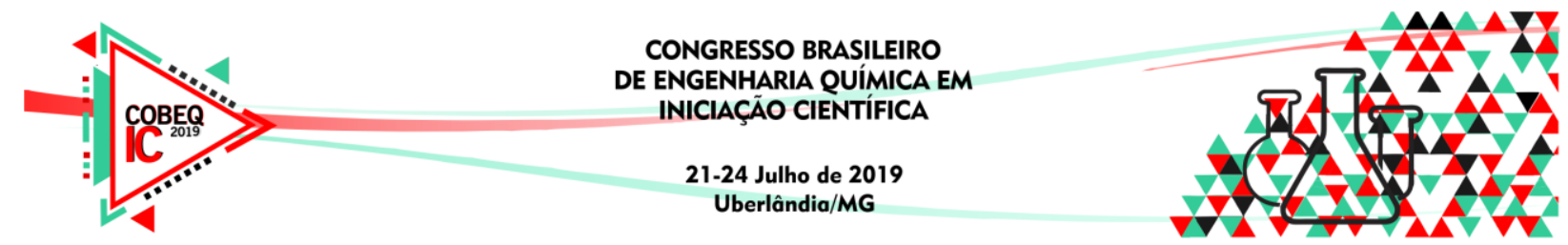

\title{
ESTUDO DO ESCOAMENTO ENTRE PLACAS EM UM TROCADOR DE CALOR UTILIZANDO TÉCNICAS DE FLUIDODINÂMICA COMPUTACIONAL
}

\author{
M.M.FILHO ${ }^{1}$, M.V.F.BASILIO ${ }^{1}$, M.H. SILVA ${ }^{1}$ \\ ${ }^{1}$ Centro Universitário da Fundação Educacional de Barretos, Departamento de Engenharia \\ Química. \\ E-mail para contato: meneghettifilho@gmail.com
}

RESUMO - Trocadores de calor a placas consistem em um conjunto de finas e metálicas placas que promovem a transferência de calor entre fluidos através de sua superfície. O projeto desses equipamentos é feito para que o efeito da turbulência aumente a dissipação de calor na placa aumentando as trocas térmicas. A necessidade de modelar esse fenômeno, com objetivo entender melhor o processo de transferência de calor $\mathrm{e} o$ comportamento do escoamento, motivou a surgir os modelos que regem esse fenômeno. Após elaboração da geometria e tratamento da malha, foram realizadas várias simulações fluidodinâmicas e os modelos de turbulência adotados foram o modelo k- $\varepsilon$ e o modelo Shear Stress Transport (SST). Os resultados indicam regiões de maior turbulência, com variações de pressão, e também é possível visualizar o comportamento do fluido estudado. Portanto, fazendo comparações com a literatura e também com o previsto pelos modelos matemáticos, é possível afirmar que os modelos reproduziram os fenômenos da transferência de quantidade de movimento e calor, sendo possível visualizá-los graficamente.

\section{INTRODUÇÃO}

Em industrias de processos químicos, é muito comum a utilização de trocadores de calor cascos e tubos para promover aquecimento ou resfriamento de correntes (Kern, 1987). Atualmente devido a versatilidade de operação, economicidade e eficiência, os trocadores de calor a placas ou PHE (Pleat Heat Exchanger) vem sendo largamente utilizados para processos de pasteurizações entre outros de fluidos a condições severas (altas pressões e temperaturas). Por proporcionarem elevadas taxas de transferência de energia, também podem operar com baixa diferença de temperatura entre as correntes. Estes trocadores são constituídos por placas metálicas corrugadas, paralelas entre si entre as quais escoam fluidos através dos canais formados e com a vazão orientada pelas gaxetas. (Shires et al., 1994).

Nestes trocadores há baixos riscos de vazamentos internos, além disso, o elevado nível de turbulência dissipa eficientemente o calor na placa, gerando perfis menores de temperatura na região metálica, além de proporcionar menores incidências de formações de incrustações nesses sistemas térmicos (Sobrinho, 2014). As placas são caracterizadas pelas ranhuras presentes na superfície de escoamento, observadas na figura 1, estas também conduzem o escoamento e proporcionam formação do efeito de turbulência. As ranhuras elevam a formação da turbulência, diminuindo o valor do número crítico de Reynolds de 10 a 400 (Leuliet, 1987). 


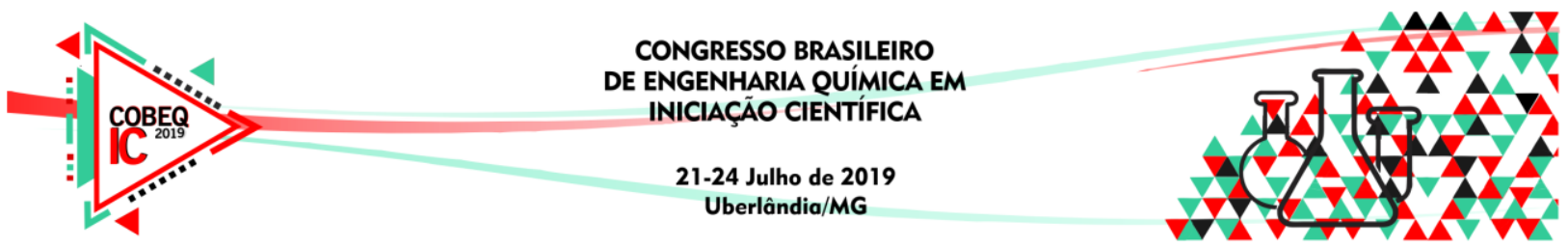

Figura 1 - Ranhuras tipo Chevron, responsáveis pela turbulência fluidodinâmica.

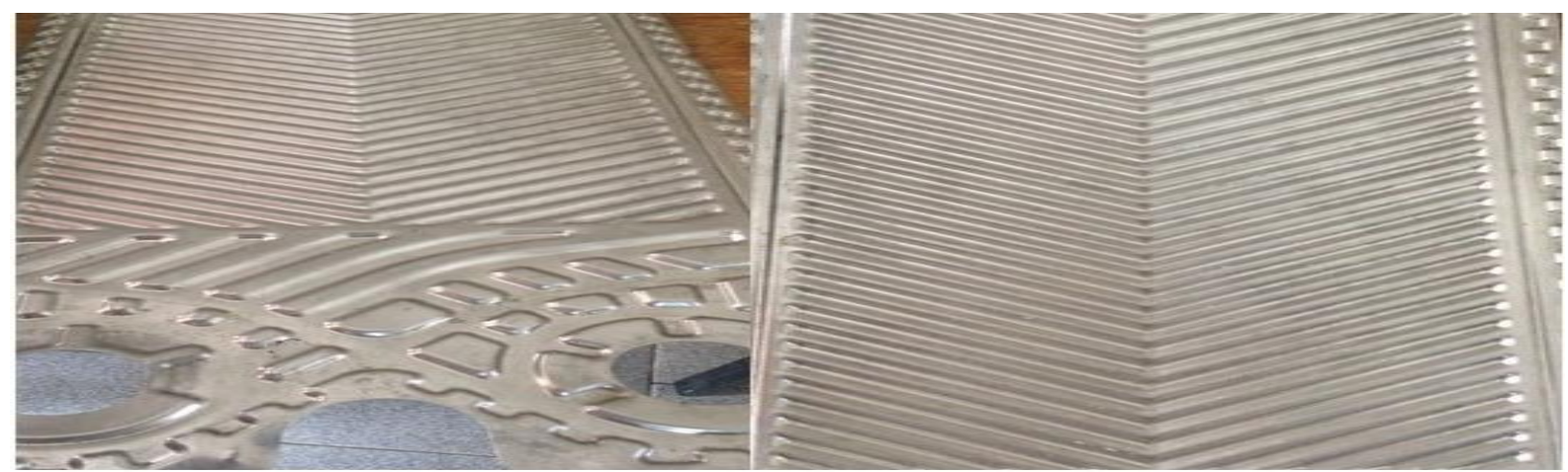

A transferência de calor ocorre na superfície da placa plana, de maneira indireta, ou seja, de um lado passa fluido frio e de outro fluido quente, através da diferente dos gradientes de temperatura, leva a troca térmica. Vários fenômenos podem transferir calor dentro do trocador, neste caso o fenômeno de convecção, definido por Incropera (2008), ocorre com fluidos em movimentos em uma superfície estática a temperaturas diferentes. Este modo de transferência de energia por convecção é mantido aleatório e pelo movimento global do fluido no interior da camada limite, camada na qual é desenvolvida pelo fluido através do movimento pela superfície aquecida em determinada região, como esta relacionada a velocidade do fluido, é denominada camada limite hidrodinâmica (Incropera, 2008).

A relação entre escoamentos e transferência de calor trata-se da quantidade de calor dissipada em determinados tipos de escoamentos, e se há variações de massa no fluido conforme movimentado. Escoamentos turbulentos são caracterizados por melhores transferências de calor e massa, devido as características do fenômeno de turbulência. Pope (2012) afirma que para aplicações na engenharia escoamentos turbulentos são predominantes, porém menos fáceis de serem observados. Em processamentos de gases ou fluidos em tubulações, compressores, os escoamentos geralmente são turbulentos, além da incrível habilidade de transportar e misturar fluidos é muito mais fácil e eficiente que em regimes laminares (Pope, 2012).

Em regimes de elevada turbulência, as partículas se apresentam em movimento aleatório, flutuações intermitentes, as camadas fluidodinâmicas invadem a direção de escoamento da camada paralela, formando escoamentos tridimensionais, vortex e dissipações de energia (ESSS, 2016). Devido ao perfil caótico, a velocidade e pressão mudam continuamente com o tempo, onde este perfil é atingido ao se ultrapassar certo valor regido pelo número de Reynolds. (Versteeg e Malalasekera, 1995). Afirmam também, que acima deste número critico de Reynolds, uma série de complicados eventos acontecem e eventualmente levam a mudança radical das características. Para aplicações na engenharia, há vários modelos que regem este tipo de escoamento, desde simples escoamentos em tubulações até complexas misturas em reatores. Para a modelagem deste fenômeno faz-se uso de vários mecanismos matemáticos que auxiliam na resolução desses problemas. Versteeg e Malalasekera (1995), reportam sobre as equações governantes para escoamentos de fluidos, apontando algumas hipóteses, entre elas a conservação da massa. Neste estudo foi considerado a conservação da massa $(\partial \rho / \partial t=0)$ e fluido Newtoniano.

\section{MATERIAIS E MÉTODOS}




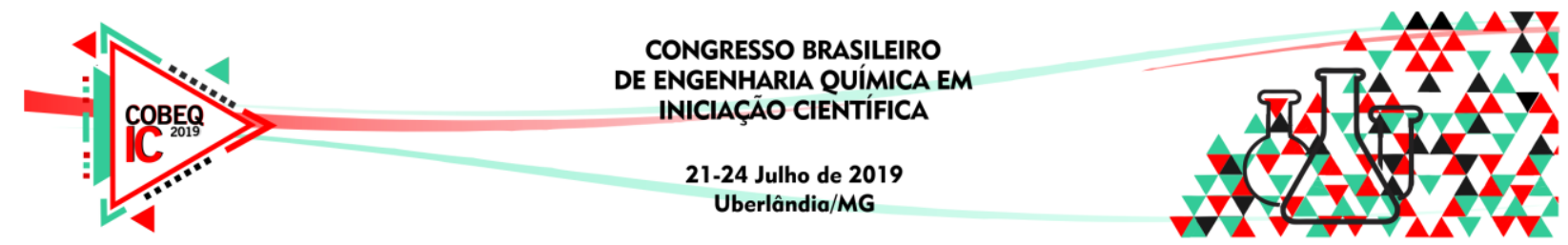

Para o estudo foi utilizado o pacote de ferramentas da plataforma ANSYS CFX, que oferece uma ampla gama de opções para modelagem de dinâmica dos fluidos, projetos e otimização de equipamentos. O programa CFX contém códigos de fluidodinâmica computacional para solucionar os problemas CFD. Os códigos CFD são estruturados em torno de algarismos numéricos que podem resolver problemas de fluxos de fluido (Versteeg e Malalasekera, 1995). Neste trabalho, foi aplicado através do software ANSYS, o método dos volumes finitos.

Geração da Geometria: o modelo da placa térmica estudada foi gerado na plataforma CFX-Pre, de forma similar a Figura 1.

Geração da malha: as malhas criadas (Figura 2b) consistem em uma parte do volume do fluido que foi dividida em partes menores de acordo com as regiões mais críticas com base na camada limite. Sendo assim consiste na primeira parte do pré-processamento dos dados, com o objetivo de produzir uma malha de entrada, onde nela de acordo com a quantidade de nós, ou seja, quanto mais refinada a malha, mais precisão tem-se nas simulações.

Figura 2a - Modelo da placa térmica. Figura 2b: Malha gerada sobre a placa.

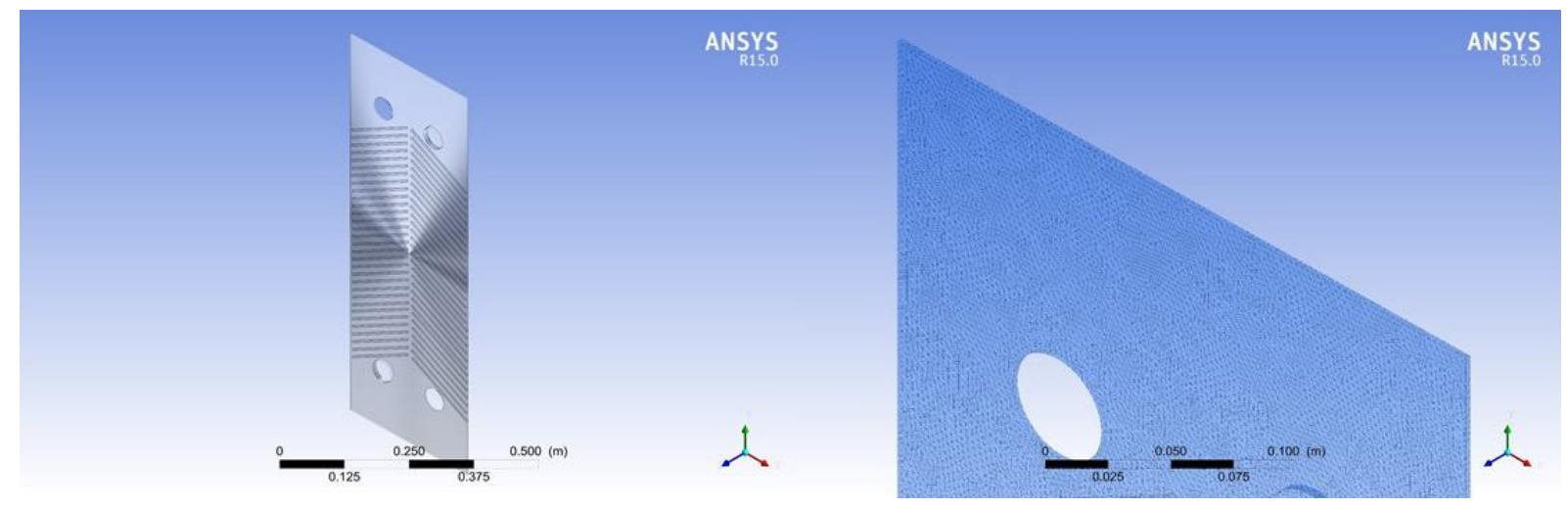

Obteve-se alguns dados quantitativos que expressam a qualidade da malha, observados na tabela 1.

Tabela 1- Características da malha nas simulações.

\begin{tabular}{|c|c|}
\hline Nós & 499.620 \\
\hline Elementos & 2.274 .299 \\
\hline Malha Métrica & Qualidade dos elementos \\
\hline Min & 0,102412042681708 \\
\hline Max & 0,999795990344573 \\
\hline Média & 0,802608096703479 \\
\hline Desvio padrão & 0,117644211295342 \\
\hline
\end{tabular}

Pré-processamento: após importar as malhas, as condições de contorno de entrada e saída e as propriedades do fluido, foram definidas assim como os modelos físicos que foram analisados, também foram definidos. Neste estudo foi abordado a modelagem dos modelos K$\varepsilon$ e SST (Shear Stress Transport).

Partindo da ferramenta dos volumes finitos e por meio da discretização das equações dos modelos propostos o software solucionou o problema fluidodinâmico. Algumas 


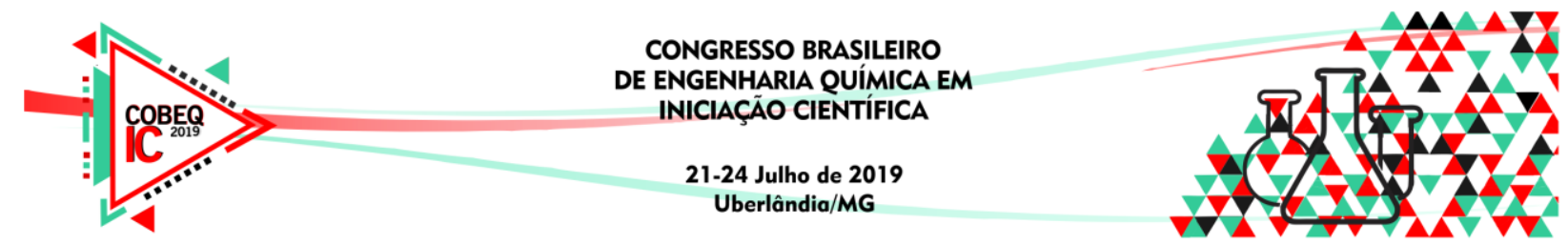

características dos modelos turbulentos são apresentadas a seguir assim como as equações que os regem.

$\boldsymbol{K}-\varepsilon$ : Versteeg e Malalasekera (1995) descrevem o modelo como o mais utilizado para casos gerais na engenharia, para descrições dos fenômenos de turbulência. Neste modelo há duas equações de transporte, uma para energia cinética turbulenta $K$ e outra para a taxa de dissipação de energia cinética $\varepsilon$, trata-se de um modelo que foca nos mecanismos que afetam a energia cinética turbulenta. As equações diferencias parciais abaixo são discretizadas para modelos de equações algébricas que podem ser solucionados numericamente, através dos processos iterativos proposto pelo software. As equações de transporte resumidamente, são apresentadas para a energia cinética $\mathrm{K}$ e para a taxa de dissipação $\varepsilon$, pelas equações 1 e 2 , respectivamente.

$$
\frac{\partial(\partial \rho \mathrm{k})}{\partial \mathrm{t}}+\operatorname{div}(\rho k U)=\operatorname{div}\left[\frac{\mu t}{\sigma k} \operatorname{gradk}\right]+2 \mu t E \mathrm{ij} \cdot E \mathrm{ij}-\rho \varepsilon
$$

$\frac{\partial(\rho \varepsilon)}{\partial t}+\operatorname{div}(\rho \varepsilon U)=\operatorname{div}\left[\frac{\mu t}{\sigma \varepsilon} \operatorname{grad} \varepsilon\right]+C \imath \varepsilon \frac{\varepsilon}{k} \mu t$ Eij.Eij$C 2 \varepsilon \rho \frac{s^{2}}{k}$

SST (Shear Stress Transport): Bardina et al., (1997), afirma que o modelo modifica a função da viscosidade turbulenta para melhorar a previsão de fluxos separados. A formulação deste modelo teve de base experimentações físicas com objetivo de prever soluções dos problemas típicos de engenharia. Trata-se de um modelo amplamente usado e robusto que combina o modelo de turbulência K- $\varepsilon$ e o modelo de K- $\omega$, amplamente usado nas regiões internas da camada limite. Para este modelo também compreendem duas equações que combinam a equação da cinética turbulenta $(K)$ e outra para a taxa de dissipação especifica de energia cinética turbulenta $(\omega)$, respectivamente as equações 4 e 5.

$$
\begin{aligned}
& \frac{\partial}{\partial \mathrm{t}}(\rho \mathrm{k})+\frac{\partial}{\partial \mathrm{xj}}\left[\left(\mu+\frac{\mu \mathrm{i}}{\sigma \mathrm{k}}\right)+\frac{\partial \mathrm{k}}{\partial \mathrm{xj}}\right]+\mathrm{Pk}-\beta \rho \omega \mathrm{k}+\mathrm{Pkb} \\
& \frac{\partial}{\partial \mathrm{t}}(\rho \omega)+\frac{\partial}{\partial \mathrm{x}}\left[\left(\mu+\frac{\mu \mathrm{t}}{\sigma \omega}\right) \frac{\partial \omega}{\partial \mathrm{xj}}\right]+\alpha \frac{\omega}{\mathrm{k}} \mathrm{Pk}-\beta \rho \omega^{2}+\mathrm{P} \omega \mathrm{b}
\end{aligned}
$$

As figuras $2 \mathrm{a}$ e $2 \mathrm{~b}$ representam respectivamente, o modelo da placa gerada para as simulações assim como a distribuição das malhas que foram geradas pela superfície da mesma. A malha gerada se encontra de forma refinada, com vários pontos de nós computacionais, que se localiza no centroide de cada volume de controle, neste ponto são calculados os valores das variáveis, ou seja, foi realizado uma análise quantitativa das equações de modelo nos pontos nodais.

Solver: foi utilizado o software CFX-solver, ferramenta que resolve as equações algébricas e gerou os resultados que foram obtidos através de um processo iterativo.

Pós-processamento: foi o componente utilizado para analisar, visualizar e apresentar os resultados de forma interativa. Neste trabalho foi possível obter a geometria da placa com as malhas de volume de controle criadas, assim como o efeito de cada modelo escolhido para os testes.

\section{RESULTADOS E DISCUSSÕES}




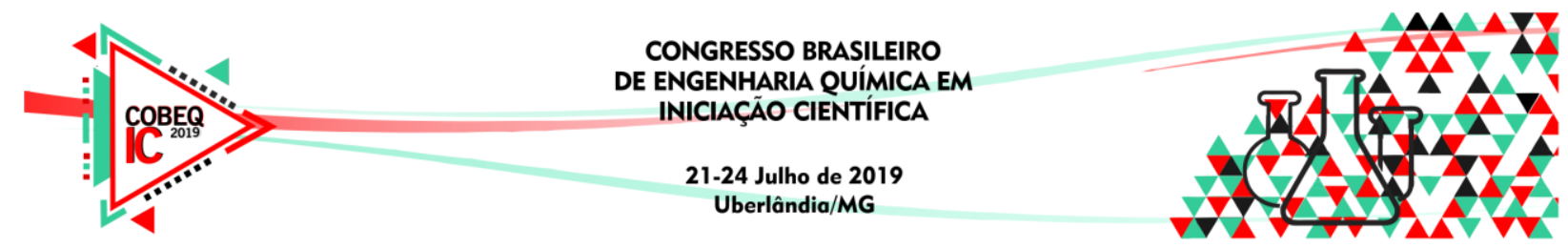

As analises na transferência de calor sobre a placa demonstram similaridade que varia de $80^{\circ} \mathrm{C}$ até $10^{\circ} \mathrm{C}$ nos dois modelos de turbulência, isso pode ser observado nas figuras $3 \mathrm{a}$ e $3 \mathrm{~b}$, em relação ao fluxo vetorial do gradiente de temperatura. Em relação ao fluido, foi estabelecido as hipóteses de que seja incompressível, assim descarta-se a possibilidade variações na massa, e que o sistema esteja em regime permanente.

Figura $3 \mathrm{a}$ - Gradiente de temperatura no modelo K- $\varepsilon$. Figura $3 \mathrm{~b}$ - Gradiente de temperatura no modelo SST.
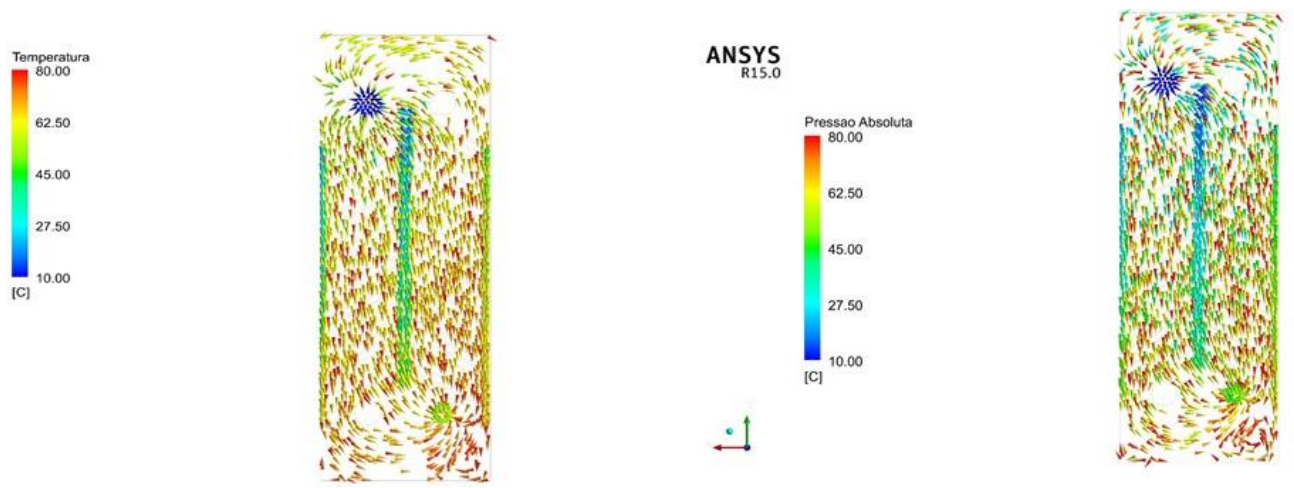

ANSYS

O modelo SST (Figura 3b) apresentou maior distribuição na transferência de calor, ao analisar a direção dos vetores, que se distribuem melhor pela placa, além disso, pelas cores azuladas indicam que ao decorrer do processo estão ocorrendo trocas térmicas entre água quente e água fria de maneira eficiente. Já o modelo $\mathrm{K}-\varepsilon$ as trocas térmicas ainda estão correndo, observados pela coloração amarelada por toda superfície. Para os mesmos modelos, as variações no gradiente de pressão também foram similares, diferenciando-se apenas nos valores das pressões de entrada, que para o modelo K- $\varepsilon$ da figura $4 \mathrm{a}$, com valor de 435334,3 Pa e para o modelo SST, o valor de 414450,47 Pa.

Figura 4a - distribuição do gradiente de pressão no modelo K- $\varepsilon$. Figura $4 \mathrm{~b}-$ Distribuição do gradiente de pressão no modelo SST.
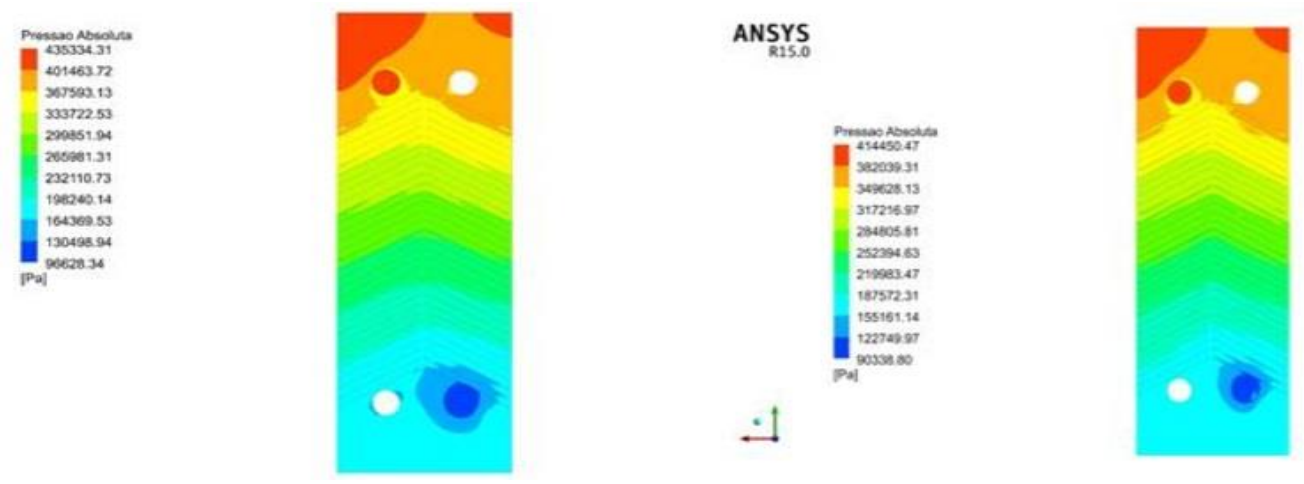

ANSYS

\section{CONCLUSÃO}




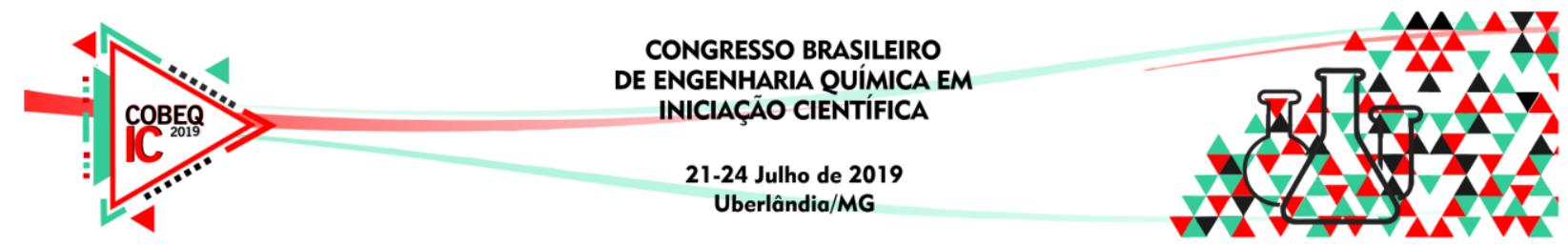

O estudo em questão possibilitou visualizar melhor os efeitos da turbulência nas placas do trocador, expondo as características do processo relacionados a pressão e a temperatura, em dois modelos de turbulência, cada um com sua equação de transporte governante. Os dois modelos testados não apresentaram diferenças gritantes nos escoamentos, porém essa observação só foi possível através dos dados gerados pela simulação computacional. Desta forma se trata de uma ferramenta para facilitar o entendimento de certos processos que são difíceis de enxergar a olho nu, representando uma opção na engenharia mais econômica, rápida e fácil para projetar, sendo possível inserir o problema fluidodinâmico em questão. Portanto neste estudo foi apresentado como são possíveis as simulações computacionais e a interpretação dos dados gerados, para diferentes modelos fluidodinâmicos.

\section{REFERÊNCIAS}

Incropera FP, Dewitt DP. Fundamentos de transferência de calor e massa. 6a Ed. Rio de Janeiro: LTC; 2008.

KERN, D. Processos de Transmissão de Calor. Rio de Janeiro: Editora Guanabara Koogan, 1987.

Leuliet, J.C., Maigonnat, J.F., Lalande, M., 1987. Etude de la perte de charge dans de échangeurs de chaleur à plaques traitant des produits non-newtoniens. Revue Générale de Thermique.

Pope S.B. Turbulent Flows. Cambrigde University Press; 2012

SHIRES, G.L.; BOTT, T.R.; HEWITT, G.F. Process Heat Transfer. Londres: Editora CRC Press, 1994.

Sobrinho G.T. Simulador de um trocador de calor de placa para um sistema de resfriamento em um reator de pesquisa[dissertação]. Rio de Janeiro: PPGIEN/CNEN: Pósgraduação em Ciências e Tecnologias Nucleares do Instituto de Engenharia Nuclear da Comissão Nacional de Energia Nuclear; 2014.

Versteeg HK, Malalasekera W. An introduction to computational fluid dynamics - the finite volume method. London: Longman; 1995. 